\title{
Entre cohérence et cohésion : humain ou performant? L'organisation dans une perspective éthique
}

\author{
Fabien Faul ${ }^{\mathrm{a}}$
}

RÉSUMÉ. La question éthique dans les organisations est indissociable de celle de la qualité des relations humaines et de la question de l'anthropologie philosophique. Penser le travail social suppose ainsi d'intégrer la réflexion menée dans et autour de la philosophie du caring, au nom d'une vulnérabilité constitutive de l'être humain. Cette perspective peut être éclairée et enrichie par la philosophie du sujet transcendantal développée au sein de la phénoménologie. La vulnérabilité, tout autant que l'appel à la responsabilité ou la réciprocité dans la vie sociale s'enracinent dans cette transcendantalité du sujet, et peuvent s'exprimer dans la narration de soi. Il y a là des dimensions que l'intégration du Don dans la vie sociale peut signifier et honorer. Dans la mesure où le travail social suppose l'accueil et la reconnaissance des sujets en tant que sujets une perspective éthique ricœurienne attirera l'attention sur la fécondité que l'on peut attendre d'une conception du sujet en termes de Soi transcendantal, de l'identité narrative ainsi que du Don.

\begin{abstract}
$\boldsymbol{A B S T R A C T}$. Ethics in organizations cannot overview the question of the quality of human relationship within a philosophical anthropology. Thinking social work leads inevitably to integrate what has been developed in and on the philosophy of "caring" and the inherent vulnerability of the human being. This prospect might be enhanced by the philosophy of the transcendental subject developed in the phenomenological tradition. $V$ unerability as well as the claim to responsibility and reciprocity in social life are rooted in that transcendental character of the buman subject and they might be expressed in a personal story to tell. Integrating Gift in social life may also express and honor that same transcendental dimension of the buman being. Given that social work implies the welcoming and the recognition of subjects as subjects, Paul Ricour's ethical prospect will show the fertility of that conception of the subject as a transcendental Self, of narrative identity and Gift.
\end{abstract}

\section{Introduction}

Aborder une question sous l'angle éthique, c'est s'intéresser à la justesse des relations humaines en jeu dans cette question. Les notions de cohérence, de cohésion, de performance, d'humanité contiennent des modèles de relations humaines potentiellement contradictoires. Une entreprise se structurera sans doute autour d'un projet de rentabilité, donc de productivité et de performance, mais ce n'est pas pour autant que sa cohésion et sa cohérence seront garanties, si pour ce faire, elle met les collaborateurs en concurrence, dans une course à la performance et à l'efficacité. Dans une telle culture d'entreprise, le questionnement éthique portera-t-il notamment sur la prise en compte des limites des personnes? Dans le milieu du travail social, on sera certainement plus attentif d'emblée aux limites des personnes, en visant un mode de relation qui «fasse preuve d'humanité », le questionnement éthique portera sur ce que veut dire concrètement : «être humain ». En quoi l'exigence de performance est-elle perçue comme contradictoire avec l'« humain »? Dans la réflexion éthique des décennies passées, cette opposition a été conceptualisée avec l'opposition entre autonomie et vulnérabilité. Cette opposition constituera notre porte d'entrée à la question de "l'organisation dans une perspective éthique ». Inévitablement la réflexion est portée vers la nécessité de clarifier de quel sujet humain l'on parle, c'est-à-dire la question de l'anthropologie, envisagée comme de domaine de la philosophique qui traite de ce qu'est, être humain. Ce sera l'objet de la seconde partie. Enfin, pour en revenir

a Maitre de conférences, Université de Lorraine 
au questionnement éthique, la troisième partie s'emploiera à revisiter l'impératif catégorique kantien, fondement de la morale moderne, et cherchera à élargir la perspective avec l'apport à cette réflexion de la question du don et de la sagesse pratique, sur les traces de Paul Ricœur.

\section{Autonomie et vulnérabilité dans la réflexion éthique contemporaine}

L'opposition entre autonomie et vulnérabilité s'inscrit dans la Modernité - entendons par là, la culture globale occidentale, qui, depuis les XVII et XVIII ${ }^{e}$ siècles véhicule les modes relationnels que nous adoptons, à l'égard des autres, du monde environnant et de nous-mêmes - et plus précisément, cette opposition s'inscrit dans une critique d'un certain nombre de tournures que prend la Modernité depuis les dernières décennies du $\mathrm{XX}^{\mathrm{e}}$ siècle. Nous commencerons par donner une vision de synthèse de ce contexte de Modernité et la critique qui en est formulée par les tenants d'une «éthique de la vulnérabilité » avant d'en énoncer quelques incidences sur la réflexion quant aux organisations.

\subsection{Le sujet moderne et son autonomie}

Percevant la nécessité de renouveler la pensée philosophique, René Descartes (2011) cherche sur quelle base nouvelle et incontestable il pourrait fonder sa pensée. C'est ainsi qu'il en vient à énoncer la seule certitude qui lui semble incontestable : lui-même existe, puisqu'il se perçoit en train de penser. Ce faisant, il introduit dans la philosophie occidentale l'idée que le sujet humain doit être conçu avant tout comme un sujet caractérisé par la faculté de penser rationnellement. Dès lors, pour les penseurs modernes, le sujet humain est pleinement humain lorsqu'il pense rationnellement, et lorsque cette rationalité lui permet de ne pas être le jeu de ses passions (Descartes, 1998). Descartes est donc l'inventeur (au sens archéologique de mettre à jour) de la pensée critique, qui consiste à ce que toute affirmation soit considérée comme vraie quand elle a été établie rationnellement comme telle, c'est-à-dire démontrée par des arguments. Le sujet moderne cartésien est autonome dans sa pensée, par son exercice rigoureux de la rationalité.
Deux siècles après Descartes, Emmanuel Kant analyse les processus de la pensée et les processus de l'agir (Kant, 2003) et considère que l'autonomie du sujet quant à ses décisions se définit comme sa capacité de se donner à soi-même les normes de son propre agir. Ceci, non pas par soumission à ses envies passagères, ni à une loi que lui dicterait une autorité extérieure à lui, ni même à un projet de bonheur mais en vertu de la capacité de la raison dite pratique (qui réfléchit en vue d'agir) de formuler rationnellement et de manière critique, des normes objectives du comportement. C'est ainsi que Kant est l'auteur de référence pour le thème de l'autonomie en référence à une philosophie du sujet rationnel.

Dans la culture moderne telle qu'elle s'est déployée depuis le XVIII ${ }^{e}$ siècle, l'autonomie du sujet est devenue l'une des valeurs les plus partagées dans ce qu'il convient d'appeler l'éthos, c'est-à-dire l'ensemble des valeurs communément admises dans la culture d'un groupe ou d'une société. L'autonomie est un projet anthropologique pour le sujet moderne, qui par les progrès de la science et de la technique ainsi que par les décisions désire prendre en main son destin et forger lui-même son humanité de demain. Sur le plan individuel, l'autonomie désigne le plus souvent la capacité d'initiative et d'action d'un sujet, comme dans le milieu médical, lorsque l'on vise à permettre à un patient la plus grande autonomie possible.

C'est ce projet d'auto-construction du sujet au nom de ce qu'on pourrait appeler avec Nietzsche une « volonté de puissance », au sens d'une volonté continue de dépassement de soi (Nietzsche, 2006), que les penseurs de la vulnérabilité ont mis en question.

\subsection{L'éthique de la vulnérabilité : une contestation du sujet moderne}

Dans les débats éthiques contemporains, l'« éthique de la vulnérabilité » a émergé comme une critique de cette conception anthropologique de l'autonomie du sujet dans le cadre de la pensée féministe américaine notamment avec Nel Noddings (1984) ou Carol Gilligan (2008). Pour les tenants de cette « éthique de la vulnérabilité », une société qui exalte l'autonomie laisse souvent pour compte les personnes plus vulnérables; il s'agit d'intégrer dans l'anthropologie et la philosophie sociale cette dimension de la vulnérabilité et de penser une réponse à la vulnérabilité pour ce qui est du care, du prendre soin d'autrui. Si, au point de départ 
de leur réflexion, ces auteures ont revendiqué une capacité féminine plus grande de prendre soin des autres, de tenir compte de leur vulnérabilité et de faire preuve de sollicitude, cette revendication a été formulée comme une exigence éthique et anthropologique pour les sociétés contemporaines. « Définir l'humain comme capable de se préoccuper des autres dans le besoin revient à reconnaître une vulnérabilité fondamentale contre la croyance en un individu toutpuissant, indépendant et détaché des relations sociales sur le mode de l'entraide ou d'une interdépendance responsable. » (Brugère, 2011, p. 52). L'« éthique du care» ne se présente donc pas comme une sorte de perspective complémentaire à mettre en œuvre pour éviter les dérives et les excès d'une anthropologie commune bâtie sur l'autonomie, mais comme un antiparadigme, une autre philosophie sociale, associée à une autre anthropologie.

L'orientation dans le sens d'une philosophie sociale - avec une critique de la théorie de la justice de John Rawls (1987) - a été développée par Joan Tronto autour des nuances de la notion anglaise (to care about, to take care of, care giving, care receiving) (Tronto, 2009) et formulée dans un autre sens avec le concept de «capabilités » du penseur Amartya Sen, et repris par Martha Nussbaum dans le sens d'une recherche de «fondation des principes politiques de base qui devraient soutenir les garanties constitutionnelles » (Nussbaum, 2008, p. 110). Elle énumère donc une liste de droits que les constitutions devraient reconnaitre à tous les citoyens et qui seraient autant de possibilités ou de moyens que la société dans son ensemble devraient se mettre en devoir de fournir à chacun: vie, santé physique, intégrité physique, sens, imagination et pensée, émotions (possibilité d'une vie affective), raison pratique, affiliation (possibilité d'appartenances à des groupes où vivre une solidarité), autres espèces (vivre des relations gratifiantes avec les autres vivants), jeu, contrôle de son environnement (Nussbaum, 2008, pp. 120-123). Vue sous l'angle de la réponse à la vulnérabilité humaine, une société qui inscrirait ces capabilités dans sa constitution serait une société qui intègrerait le care.

\subsection{Quelle application de cette dialectique aux organisations}

Cette problématique de la critique du sujet autonome au nom de la vulnérabilité peut questionner l'éthique appliquée aux organisations socio-professionnelles en particulier sur leur discours managérial : présente-t-on un idéal de performance, d'efficacité, d'indépendance? Dans ce cas, quelle intégration pour les personnes vulnérables? Ceci se comprend en relation avec les représentations et idéaux de la société où les modèles les plus fréquemment mis en œuvre notamment par la publicité - sont ceux de personnes mobiles, minces, sportives, détendues à tout moment, sûres d'elles-mêmes et de leurs moyens, de leurs performances, de leur efficacité...

On peut certainement constater depuis quelques années une sensibilisation accrue aux personnes en situation de vulnérabilité, d'où les lois pour aider l'embauche de personnes handicapées, des réflexions sur la responsabilité sociale des entreprises et tout particulièrement sur leur contribution à l'élaboration et la mise en place de la vie en société, avec la prise en compte d'une impossibilité à distinguer totalement la sphère économique de la sphère politique ou sociétale. On peut évoquer également dans ce sens, une sensibilité générale pour les victimes, de quelque ordre qu'elles soient, aussi bien les victimes de catastrophes naturelles, comme le montrent les opérations de solidarité qui se mettent en place au plan mondial en cas de catastrophe naturelle, que les victimes de harcèlement au travail ou dans les familles ou les victimes de discriminations. On peut se souvenir de l'étude à ce sujet d'Eliachef et Soulez-Larivière (2007). Le sentiment du devoir de secourir les victimes constitue ainsi une application concrète du care.

Dans le cadre des organisations, se donner une charte éthique est certainement un premier pas qui permet de définir comment elles tiennent compte des vulnérabilités de ses membres et des lignes de conduite qu'elles se donnent.

Toutefois, la dialectique de l'éthique de l'autonomie et de l'éthique de la vulnérabilité peut aussi être questionnée. D’une part, cette problématique est susceptible de pâtir d'un réductionnisme par son aspect très binaire. Elle risque de laisser entendre qu'il y a d'un côté les personnes autonomes et de l'autre des personnes vulnérables dont la prise en charge constituerait une responsabilité pour les personnes dites «autonomes». Il n'y a pas de difficulté à montrer que chaque sujet a sa part d'autonomie et sa part de vulnérabilité. 
On peut déplorer également une lacune quant à la définition de la vulnérabilité : les auteurs qui en traitent utilisent souvent les concepts de fragilité, de finitude, ou de dépendance comme des synonymes de la vulnérabilité. On peut citer Ricœur (2001), Ferrarese (2009) ou Valadier (2011). Toutefois il est toujours possible de trouver d'autres formes de vulnérabilité (précarité, passibilité, contingence, faillibilité, mortalité, etc.) sans compter la distinction des formes physiques et des formes psychiques de la vulnérabilité, désignées souvent par des notions prises au sens figuré à l'instar du mot vulnérabilité lui-même, qui désigne littéralement la possibilité d'être blessé physiquement. Or la notion de blessure est souvent prise au sens figuré, lorsqu'elle désigne une offense, un sentiment d'avoir été « atteint » par une attitude ou une parole. On pourrait en dire autant de la fragilité, qui désigne la possibilité pour un objet d'être brisé, etc. Tout ceci fait en sorte que le contour du concept de vulnérabilité reste souvent flou. D’une certaine façon, on pourrait proposer une perspective globale en considérant que la vulnérabilité désigne l'expérience du caractère non-absolu de la condition humaine. Ceci pourrait être confirmé si l'on envisage l'autonomie comme une forme d'illusion de toutepuissance comportant une prétention fantasmatique d'être absolu.

N'existe-t-il pas une forme de dissymétrie entre l'autonomie et la vulnérabilité? D'une part, parce que le plus souvent un sujet prend conscience de sa vulnérabilité à l'occasion de situations très particulières (accidents, épreuves de santé, épreuves relationnelles, etc.) qui lui indiquent qu'il est vulnérable, alors qu'un fantasme archaïque de toute-puissance susceptible de lui inspirer une certaine idée de l'autonomie est toujours présent dans le fond de son psychisme. Cellesci, l'autonomie et la vulnérabilité, ne sont pas des réalités si évidentes qu'il n'y parait : elles doivent être élaborées, c'est-à-dire pensées, verbalisées, définies dans un travail critique. Cependant, la première appelle une régulation, la seconde, un consentement. Ensuite, si le care est la réponse à la vulnérabilité, fautil viser à ce qu'une personne puisse sortir de sa situation de vulnérabilité, et auquel cas aller vers une autonomie? Et si c'est le cas, laquelle? Peut-on envisager qu'elle a une marge d'évolution suffisante pour sortir d'une situation particulière de vulnérabilité ou bien faut-il envisager de l'aider à l'« assumer »? Dans ce cas, que veut dire « assumer une vulnérabilité »: s'y résigner? Rebondir? Entrer dans un processus de résilience?

\section{Le point de vue d'une philosophie du sujet : du sujet cartésien au sujet ricoeurien}

Si la dialectique de l'autonomie et de la vulnérabilité peut représenter un premier éclairage sur l'éthique dans les organisations, tentons une seconde approche par une autre porte d'entrée : la philosophie du sujet.

\subsection{La pensée cartésienne, creuset du sujet autonome moderne}

Dans les Méditations métaphysiques, René Descartes (2011) ambitionne de refonder la philosophie sur une base certaine : il le fait dans un itinéraire réflexif, mettant en scène (dans une situation heuristique) le doute systématique. Cette démarche le conduit à une affirmation incontestable, car elle procède de l'évidence : la seule réalité certaine est l'expérience de soi-même pensant, et capable de penser de manière critique, en vérifiant si ce que l'on pense repose sur des fondements évidents. Ce faisant, la pensée de Descartes a été reconnue comme le moment-fondateur de l'anthropologie philosophique moderne, caractérisée par sa rationalité, laquelle se développe avec rigueur dans la science et la technique. Pour les philosophes de la vie sociale des XVII et XVIII siècles, la vie en société procède également d'une démarche rationnelle, décidée (le contrat social), en fonction d'intérêts bien calculés. Le contrat social repose sur le raisonnement selon lequel on a davantage intérêt à vivre en société en acceptant les contraintes inévitables que cette organisation génère, qu'à ne pas s'organiser.

Les $\mathrm{XIX}^{\mathrm{e}}$ et $\mathrm{XX}^{\mathrm{e}}$ siècles ont vu se déployer cette anthropologie dans une société qui s'est technicisée, industrialisée et qui a développé des projets politiques censés assurer le progrès de l'humanité. En raison d'un certain nombre de déconvenues plus ou moins traumatisantes (guerres, crises économiques, catastrophes sanitaires ou écologiques), la période contemporaine nous voit dans un état global de précarité, de vide de sens, d'angoisse devant un avenir dont nous doutons à présent qu'il soit un réel progrès. Parmi les auteurs qui ont mis en exergue ces évolutions, on peut citer Gilles Lipovetsky (1983), Olivier Rey (2006) ou encore Alain Ehrenberg (2010). Dans ce contexte culturel, on pourrait re-visiter la notion de mélancolie, audelà de la définition classique généralement admise, 
de prédisposition à la tristesse, plus ou moins teintée de complaisance (voir le Littrê). Si notre environnement scientifique, technique et politique est potentiellement à l'origine de drames alors qu'il est censé mener au progrès, nous nous sentons confusément dans une impasse : que nous voulions ou que nous renoncions à une perspective de progrès, que nous voulions ce progrès par le moyen de la science, de la technique et de la politique modernes, ou que nous y renoncions, nous sommes susceptibles d'être confrontés à des drames. C'est le malaise engendré par cet état de fait que l'on pourrait appeler mélancolie, entendue comme une conscience aiguë du tragique de l'existence.

La rationalité n'étant plus ce qui enchante l'anthropologie actuelle, on s'aperçoit que la culture présente demande à l'affectivité, ou à l'émotion, d'être le lieu qui nous indique que nous sommes un sujet. Si l'on veut bien considérer que nous sommes en train de passer de la modernité vers une " post-modernité », le sujet postmoderne se caractérise par la subjectivité émotionnelle : il est ce qu'il ressent qu'il est, dans un ici et maintenant souvent amnésique et sans souci de l'avenir.

On peut remarquer que l'art est le premier domaine où il a été question de post-modernité, dès les années soixante, constatant que l'art avait emboîté le pas à Marcel Duchamp (Lipovetsky, 1983) dans la postérité de qui l'art n'est plus une question de beau ou de savoir-faire, mais on considèrera comme une œuvre d'art, ce que l'artiste a décidé que cela le serait, parce qu'« il le sent » comme cela à tel instant précis. On attend de l'art qu'il procède et qu'il suscite de l'émotion.

D’une certaine manière, cette pensée reste dans la perspective d'une autonomie, qui serait une « autonomie émotionnelle », puisqu'il s'agit de tenir compte du « ressenti » de chacun et que ce ressenti est normatif pour lui, et qui ne saurait être mis en question par d'autres, qui «ne le sentent pas » de la même façon. L'agir n'est pas pensé comme la mise en œuvre d'une maxime - comme chez Emmanuel Kant - mais comme la mise en œuvre de l'impulsion intérieure ou de l'émotion du moment.

Si l'humanité se définit par des sensibilités, le modèle de vie sociale que cette anthropologie génère est celui d'une vie sociale sous-tendue par des affinités affectives, des sensibilités ou des manières communes d'envisager le sens de l'existence. En somme, une société de type communautarien - étant entendu qu’à l'instar d'Alasdair MacIntyre, les penseurs du communautarisme ne se situent pas purement et simplement sur le registre d'une affinité spontanée, mais la communauté unificatrice est également l'objet d'une élaboration raisonnée (MacIntyre, 2006).

En réponse à ces problématiques, Paul Ricœur (1990) entend unifier les multiples dimensions des relations humaines : la relation à soi et la représentation que l'on se fait d'une vie accomplie (le « je »), les relations interpersonnelles (le « tu »), et la relation à l'ensemble de la société par la médiation des institutions (le «il»). Le sujet humain ne peut être délié de la vie en société, mais cela suppose aussi qu'il ait une «visée de la vie bonne », personnelle et sociale (Ricœur, 1990).

\subsection{Une philosophie du sujet transcendantal}

Critiquant, dans ses Méditations cartésiennes, la pensée de René Descartes faisant du cogito le fondement de sa philosophie du sujet, Edmund Husserl (2001) élabore l'idée d'un "sujet transcendantal», désignant par là le fait qu'il ne s’identifie à rien de ce qu'il peut connaître de lui et à rien de ce qu'il peut dire de lui-même; le sujet s'expérimente toujours plus vaste que tout ce qui peut le qualifier. Il accède à cette réalité en mettant entre parenthèses tout ce qu'il peut affirmer ou connaittre (l'épochë) pour se rendre compte qu'il est « horizon d'intentionnalité » (Husserl, 2001). D'une manière indissociable, le sujet a besoin des qualificatifs, ou des prédicats - qu'il reçoit des relations interpersonnelles - pour s'auto-définir et prendre conscience du fait d'être un sujet, et c'est ce faisant, qu'il éprouve sa propre transcendance, en vertu de laquelle il ne s'identifie à aucun prédicat ni à leur ensemble.

Paul Ricœur désigne le sujet dans sa singularité transcendantale comme un " insubstituable », et il reprend à Gilles Gaston Granger la notion d'« ancrage » qui « renvoie à une position non substituable, à un unique centre de perspective sur le monde » (Granger, 1979 cité dans Ricœur, 1990, p. 65). En parlant d'«aporie de l'ancrage », il désigne la non-coïncidence entre d'une part l'expérience de soi comme sujet transcendantal, irréductible à toute prédication (ancrage) et d'autre part, la désignation de soi par des catégories, par exemple celles de l'état civil (Ricœur, 1990, p. 68). Cette expérience d'un Soi unit à la 
fois un « pouvoir dire » et une impossibilité à pouvoir se dire totalement. On pourrait rapprocher ce paradoxe du second moment de son Parcours de la reconnaissance, qui est une "reconnaissance de soi-même » (Ricœur, 2004).

L'expérience du Soi comme sujet transcendantal comporte donc inévitablement non seulement le constat de cette transcendance, mais l'attente d'une reconnaissance par les autres de cette subjectivité transcendantale. Cela peut s'entendre dans la relation interpersonnelle et dans la relation en société. S'expérimenter comme un Soi transcendantal comporte l'attente d'être accueilli par d'autres comme tel, et d'occuper une place personnelle - insubstituable dans un réseau de relations.

Ici, l'on rencontre la troisième forme de reconnaissance, selon Ricœur : la reconnaissance "réciproque » - qu'il exprime dans Soi-même comme un autre comme une «réciprocité des insubstituables». On peut remarquer que cela vaut non seulement dans une réciprocité du «je-tu», mais aussi dans une dynamique du « je-nous ».

Si tant est que le sujet transcendantal est porteur de cette attente de reconnaissance, il fait aussi l'objet de deux menaces possibles ou, formulé autrement, qu'il est possiblement hanté par deux causes d'angoisse: d'une part, la menace d'être identifié à un prédicat et d'autre part, de ne pas se voir reconnaitre une place dans les relations. Conscient ou non d'être plus vaste que tout ce qui peut le qualifier, le sujet éprouvera un malaise chaque fois qu'il est identifié à un aspect de lui, dans les relations humaines, même lorsque cet aspect est exact mais néanmoins partiel; un sujet n'est pas que ce que l'on peut dire même avec justesse qu'il est. Et de la même façon, il ne se sent pas reconnu si, dans les relations humaines, l'on ne lui signifie pas qu'il est porteur de cette transcendance, en termes ricœuriens, si l'on ne le met pas en situation de « réciprocité des insubstituables». Dans les relations sociales, une telle non-reconnaissance peut générer deux réactions contradictoires : soit la crainte d'une fusion dé-personnalisante dans le groupe, soit la nécessité d'une lutte pour la reconnaissance.

La plainte, la révolte, le cri, sinon la réaction violente peuvent alors être décryptés comme le symptôme d'une telle non-reconnaissance et la réaction qui réclame une reconnaissance du caractère transcendantal dont le sujet fait peu ou prou l'expérience qu’il est constitué.
En définitive, la possibilité de ne pas être reconnu dans les relations humaines comme un Soi est peut-être la première vulnérabilité dont tout sujet est porteur.

\subsection{Le sujet ricoeurien : l'identité narrative}

Sur la question du sujet, Paul Ricœur propose une autre piste : le sujet ricœurien est celui qui dit: «je peux », et en particulier : «je peux me raconter»-le troisième pouvoir de la reconnaissance de soi-même dans le Parcours de la reconnaissance (Ricœur, 2004, p. 150). Il suggère par là que la meilleure façon de donner à sentir comment un sujet se perçoit comme un Soi est d'entendre ce qu'il dit de lui lorsqu'il se raconte. En d'autres termes, le sujet narratif dit quelque chose de lui mais en dit plus de lui par la narration que ce qu'il pourrait dire de lui par une autoqualification. Pour Ricœur, et du point de vue de l'éthique, la narration a la capacité de dépasser une dichotomie fondatrice dans la morale moderne et énoncée par Hume : l'opposition de l'énonciation et de la prescription, et considérant que d'un être (d'un état de fait) on ne saurait tirer un devoir-être.

Du point de vue de cette identité narrative, on touche une autre figure de la vulnérabilité : elle est éprouvée lorsque dans certaines situations particulières, comme sujet, l'on n'est pas entendu dans ce que l'on dit de soi, et que l'on est réduit à un ensemble de représentations de soi ou de l'autre, lorsque ce que quelqu'un croit savoir d'un sujet procède en fait d'une non lucidité sur lui-même, de projections, etc.

Concernant les organisations, cette question conduit à se demander quelle est la part accordée à l'histoire de l'organisation et à l'histoire des personnes qui en sont partie prenante. Par ailleurs, on peut aussi se demander quel souci une organisation liée au travail social développe pour permettre à des personnes en situation de vulnérabilité de se raconter, au moment de leur prise en charge.

\section{Problématiques liées à l'éthique des organisations}

Au terme de ces suggestions d'ordre anthropologique, évoquons trois problématiques liées à l'éthique dans les organisations : elles sont surtout pensées comme des réflexions heuristiques. 


\subsection{Retour vers la $2^{\mathrm{e}}$ variante de l'impératif catégorique de Kant}

La morale d'Emmanuel Kant est souvent connue pour et identifiée à la deuxième variante de l'impératif catégorique, qui lui donne un contenu: "Agis toujours de manière à traiter l'humanité aussi bien dans ta personne que dans la personne de tout autrui, toujours déjà comme une fin et jamais simplement comme un moyen » (Kant, 1994, p. 108). Si l'on entend par «l'humanité dans la personne» dans la perspective du sujet transcendantal, cela veut dire que cet impératif institue un principe de non-confusion entre le sujet et tout prédicat le concernant: ses fonctions, capacités, qualités, caractéristiques. Toute réduction d'une personne à l'un de ces aspects est déjà une manière de plus de l'envisager comme une fin en soi.

Cet impératif attire en particulier l'attention sur la non-confusion entre la personne et ses fonctions: dans les relations de travail, ce sont toujours deux personnes - deux sujets transcendantaux ineffables qui sont en relation de réciprocité et qui exercent des fonctions descriptibles. Identifier un sujet à ses fonctions, c'est prétendre l'inscrire et le circonscrire dans un registre de qualification qui est déjà une nonreconnaissance, et l'identification de l'humanité à un moyen. Lorsque l'impératif kantien dit : «jamais simplement comme un moyen ", il reconnait qu'une personne peut avoir un rang de moyen dans une organisation, en raison de la fonction qu'elle y occupe. La réduction commence lorsque l'on ne voit de la personne que sa fonction en omettant qu'en tant que sujet, elle transcende sa fonction. Ceci questionne aussi bien les relations au sein d'une hiérarchie que les relations d'une organisation avec les personnes qui ont recours à ses services. Une personne n'est jamais simplement un demandeur d'emploi, le résident d'un Établissement d'Hébergement pour Personnes Âgées Dépendantes (EHPAD), un enfant « placé », etc.

\subsection{Le don, comme reconnaissance du sujet}

Dans le Parcours de la reconnaissance, Paul Ricœur en vient aux recherches de Marcel Mauss (2007) sur le don. Marcel Hénaff reprend à son tour ces recherches pour signifier que le lien social peut être pensé sur un autre mode que le calcul d'intérêt comme le laisse entendre la notion de « contrat social». Hénaff insiste sur le fait que le don cérémoniel mis en évidence par
Mauss n'est pas un échange marchand, même si celui qui accepte le don se sent redevable et tenu de rendre, dans un contre-don estimé équivalent au don initial. D'où la structure anthropologique du donner-recevoirrendre (Hénaff, 2012).

Si la relation interpersonnelle exige l'intégration d'une dimension transcendantale du sujet humain, cette dimension relève d'une forme de "gratuité », au sens de non-évaluable, non-négociable ou de noncontractualisable : elle dépasse ou « transcende » aussi les objectifs concrets d'une organisation et tout ce qui est descriptible ou quantifiable. Une personne ne peut s'identifier à un projet, des réalisations, des bilans, si bien que le fait de tenir compte de cette dimension du Soi procède déjà du don : le don d'une réciprocité de sujets transcendantaux. Une telle réciprocité ne peut procéder d'un contrat ou d'une convention, car une relation contractuelle institue des droits et des devoirs clairement repérables. Un contrat évolue dans la sphère des fonctions et des prédicats. Une « réciprocité des insubstituables » se met en place à l'initiative d'une personne consciente de ce que l'enjeu d'une relation humaine se situe avant tout là, dans la considération du caractère transcendantal du sujet, et indépendamment d'une vérification préalable pour savoir si la personne qui lui fait face en a conscience, elle également. Dans le cas de deux personnes averties de l'enjeu relationnel de reconnaissance du caractère transcendantal du sujet, les deux ont le sentiment d'une véritable rencontre. Si dans un face-à-face, un sujet n'a pas fait ce cheminement, on peut penser que le fait d'être accueilli et considéré comme un sujet transcendantal fera entrer en résonance en lui une dimension dont il est constitué, lui fera faire une expérience concrète d'une reconnaissance - même s'il peut être difficile pour lui de mettre cette expérience en mots - et ce sera probablement pour lui un jalon vers une plus grande justesse relationnelle.

\section{3 Éléments d'une sagesse pratique dans les organisations}

«Sagesse pratique» est l'expression par laquelle Ricœur traduit phronèsis d'Aristote, autrefois traduit par « prudence » et dont Pierre Aubenque a synthétisé les multiples aspects (Aubenque, 2009). «La sagesse pratique, dit Paul Ricœur, est ce discernement, ce coup d'œil en situation d'incertitude, braqué sur l'action qui convient» (Ricœur, 2004, p. 135). On pourrait considérer que la sagesse pratique est la capacité de décider d'une manière appropriée, en situation; 
et une situation est une « configuration relationnelle circonstanciée $»$.

Dans une organisation, grosso modo, les éléments en présence sont :

- un objectif commun de l'organisation, fédérateur d'une union et d'une action commune, d'une solidarité;

- les apports et mises en œuvre des sujets, leurs capacités, talents (d'où leur fonction et l'instauration d'une hiérarchie);

- la présence de sujets transcendants en relation (sur les modes du « je-tu », «je-il », «jenous »);

- des valeurs qui guident un objectif commun (notamment, puisqu'il s'agit d'une organisation, l'exigence de justice);

- l'affrontement de la finitude et de la contingence du monde.

La sagesse pratique est l'attitude par laquelle chacun de ces éléments est reconnu pour lui-même, qui suppose une forme de perception de ces différents éléments et de leur importance en fonction des situations et enjeux. Et sur la base du «coup d'œil» sur cette complexité de composantes, elle est la recherche d'une prise de décision ajustée aux personnes impliquées dans une situation particulière.

C'est le plus souvent l'expérience acquise et partagée dans les organisations, le dialogue pluridisciplinaire, le fait de raconter des crises traversées qui permettent de constituer collectivement une sagesse pratique dans une organisation. La constitution collective d'une sagesse pratique est probablement l'un des grands enjeux éthiques d'une organisation, dans un monde en mutation où les personnes en situation de responsabilité ont souvent l'impression d'improviser leur décision. Musicalement, l'improvisation loin d'une création ex nibilo, met en œuvre des techniques acquises par le travail et l'expérience, pour modifier des airs connus et comme en révéler des potentialités jusque-là insoupçonnées.

\section{RÉFÉRENCES}

Aubenque, P. (2009). La prudence chez Aristote. [1963]. Paris, France : Presses universitaires de France.

Brugère, F. (2011). L'éthique du Care. Paris, France : Presses universitaires de France.

Descartes, R. (2011). Méditations métaphysiques. Objections et réponses. [1641]. Paris, France : Flammarion.

Descartes, R. (1998). Les passions de l'âme. [1649]. Paris, France : Flammarion.

Ehrenberg, A. (2010). La société du malaise. Paris, France : Odile Jacob.

Eliacheff, C. et Soulez-Larivière, D. (2007). Le temps des victimes. Paris, France : Albin-Michel.

Ferrarese, E. (2009). Vivre à la merci. Le care et les trois figures de la vulnérabilité dans les théories politiques contemporaines. Multitudes, 37-38(2) 132-141. doi.org/10.3917/mult.037.0132

Gilligan, C. (2008). Une voix différente. Pour une éthique du care. [1982]. Paris, France : Champs-Essais, Flammarion.

Granger, G.G. (1979). Langage et épistémologie. Paris, France : Klincksieck.

Hénaff, M. (2012). Le don des philosophes. Repenser la réciprocité. Paris, France : Éditions du Seuil.

Husserl, E. (2001). Méditations cartésiennes. Introduction à la phénoménologie. [1931]. Paris, France : J. Vrin.

Kant, E. (1994). Fondation de la métaphysique des mours. [1785]. Paris, France : Flammarion.

Kant, E. (2003). Critique de la raison pratique. [1788]. Paris, France : Flammarion.

Lipovetsky, G. (1983). L'ère du vide. Essai sur l'individualisme contemporain. Paris, France : Gallimard.

Maillard, N. (2011). La vulnérabilité. Une nouvelle catégorie morale? Genève, Suisse : Labor et Fides.

Mauss, M. (2007). Essai sur le don. [1923-1924]. Paris, France : Presses universitaires de France.

MacIntyre, A. (2006). Après la vertu. [1981]. Paris, France : Presses universitaires de France. 
Nietzsche, F. (2006). Ainsi parlait Zarathoustra. [1883-1885]. Paris, France : Flammarion.

Noddings, N. (1984). Caring: a Feminine Approach to Ethics and Moral Education. Berkeley, United States: University of California Press.

Rey, O. (2006). Une folle solitude. Le fantasme de l'homme auto-construit. Paris, France : Éditions du Seuil.

Ricœur, P. (1990). Soi-même comme un autre. Paris, France : Éditions du Seuil.

Ricœur, P. (2001). Le juste 2. Paris, France : Esprit.

Ricœur, P. (2004). Parcours de la reconnaissance. Paris, France : Stock.

Tronto, J. (2009). Un monde vulnérable. Pour une politique du care. [1993]. Paris, France : La Découverte.

Valadier, P. (2011). Apologie de la vulnérabilité. Études, 414(2), 199-210. 